\title{
Shear Thickening Creep in Superplastic Silicon Nitride
}

\author{
I-Wei Chen* and Shyh-Lung Hwang
}

Department of Materials Science and Engineering, University of Michigan, Ann Arbor, Michigan 48109-2136

\begin{abstract}
A novel shear-thickening phenomenon has been observed in superplastic silicon nitrides compression tested between $1500^{\circ}$ and $1600^{\circ} \mathrm{C}$. Liquid-enhanced creep of SiAlONs undergoes a transition from Newtonian behavior to shear-thickening behavior at a characteristic stress, with the strain rate sensitivity increasing from unity to around 2 . The transition stress is always around $20 \mathrm{MPa}$, even though the Newtonian flow stress is very sensitive to temperature, grain size, and phase composition. Rheopexic hysteresis, manifested as a slow stress relaxation to a steady-state value after a strain rate decrease, was also observed in the shear-thickening regime. We attribute the cause for shear thickening to a repulsive force between initially wetted SiAlON grains, which form a "dry" and "rigid" bridge in between when pressed above a characteristic stress, possibly due to the contact of the residue Stern layers on the opposing grain/liquid interfaces. A micromechanical model, which takes into account the stress variation among differently oriented grain boundaries, has been formulated to assess the effect of "rigid" grain boundaries. A continual stochastic rearrangement of grain configurations and a relatively thick Stern layer are suggested as the necessary prerequisites for shear thickening in liquid-enhanced creep. [Key words: sialons, superplastic, shear, creep, models.]
\end{abstract}

\section{Introduction}

$\mathbf{C}^{n}$ REEP, including superplastic flow of ceramic materials and metals, is commonly described by a power law

$$
\dot{\varepsilon}=\alpha \sigma^{n}
$$

where $\dot{\varepsilon}$ is the creep rate, $\sigma$ is the flow stress, $\alpha$ is a material constant, and $n$ is a nondimensional material constant. When the creep mechanism is that of diffusional plating of grain boundaries, $n$ equal to one is often found, and such flow behavior is formally analogous to Newtonian flow in fluid mechanics. On the other hand, a higher $n$ value can arise when deformation is at least partially attributed to dislocations or cavitation or is somehow limited by interface reactions. A stress exponent lower than unity has never been reported in these materials to the best of our knowledge..$^{1-2}$

Deformation of slurries, colloids, and polymeric liquids can be similarly described by Eq. (1). However, both $n>1$ and $n<1$ are commonly observed, depending on the materials. Expressed in terms of viscosity, $\eta=\sigma / \dot{\varepsilon}$, shear thinning corresponds to $n>1$ for a decreasing viscosity at a higher strain rate, while shear thickening corresponds to $n<1$ for an increasing viscosity at a higher strain rate. ${ }^{3}$ Quantitative models have been proposed for shear thickening in polymeric melts, drawing on the special features of molecular configurations

G. M. Pharr-contributing editor

Manuscript No. 196373. Received September 9, 1991; approved January 20,1992

Supported by the U.S. Air Force Office of Scientific Research under Grant No. 87-0289.

${ }^{\star}$ Member, American Ceramic Society. that evolve with shear rate. ${ }^{4.5}$ In colloids it has also been linked to either the formation of an extended gellike superstructure between particles ${ }^{6-7}$ or to the breakdown of easy-slip patterns..$^{8-9}$ On the other hand, shear thickening in slurries and granular materials, such as wet sand and soil, is generally attributed to dilatant flow involving particles rolling over each other with dewetted liquid trapped in interstices. ${ }^{10,11}$ In the latter two classes of materials (colloids and slurries), rheological studies are mostly limited to solid volume fractions of no more than $75 \%$.

Superplastic SiAlONS of very fine microstructures which exhibit tensile ductilities in excess of several hundred percent have been recently discovered. ${ }^{12}$ SiAlONS are solid solutions based on $\mathrm{Si}_{3} \mathrm{~N}_{4}$. When the tetrahedral $\mathrm{Si}-\mathrm{N}$ network is modified by substitution of $\mathrm{Al}$ for $\mathrm{Si}$ and $\mathrm{O}$ for $\mathrm{N}, \beta^{\prime}$-SiAlON isomorphic with $\beta-\mathrm{Si}_{3} \mathrm{~N}_{4}$ forms with a general formula of $\mathrm{Si}_{6-x} \mathrm{Al}_{x} \mathrm{O}_{x} \mathrm{~N}_{8-x} .{ }^{3}$ When additional metal ion interstitials are added to the network, the formula is altered to become $\mathrm{M}_{z / n^{-}}$ $\mathrm{Si}_{6-x-z} \mathrm{Al}_{x+z} \mathrm{O}_{x} \mathrm{~N}_{8-x}$, and the crystalline structure is converted to one isomorphic with $\alpha-\mathrm{Si}_{3} \mathrm{~N}_{4}$. This is termed $\alpha^{\prime}$-SiAlON. ${ }^{14}$ In most cases, some amount of glassy phase ranging from 5 to 20 vol\% coexists with $\alpha^{\prime}$ and $\beta^{\prime}$ SiAlONS. ${ }^{15}$ It is in this class of ceramics that we have observed a novel Newtonian-toshear-thickening transition which occurs at a characteristic stress, ca. $20 \mathrm{MPa}$ in compression between $1500^{\circ}$ and $1600^{\circ} \mathrm{C}$. The main experimental observation of this highly unusual phenomenon is reported in the present paper, along with a theoretical model that rationalizes the transition. Further details of the materials development, their microstructures, and their constitutive behavior has been reported elsewhere. ${ }^{\text {is }}$

\section{Experimental Procedure}

\section{(1) Materials}

SiAlONS used in this study were prepared by mixing proper amounts of $\mathrm{Si}_{3} \mathrm{~N}_{4}, \mathrm{AlN}, \mathrm{Al}_{2} \mathrm{O}_{3}$, and $\mathrm{Y}_{2} \mathrm{O}_{3}$ powders. The starting powders were very fine and, in the case of $\mathrm{Si}_{3} \mathrm{~N}_{4}$, contain a very high $\alpha$-phase content. They were attrition milled in isopropol alcohol for $2 \mathrm{~h}$ with porcelain or high-purity alumina milling media. The milled slurry was stirred and dried under heat to obtain a uniform powder mixture. Fully dense ceramic blanks were then prepared by hot-pressing under $28 \mathrm{MPa}$ for $20 \mathrm{~min}$ at $1550^{\circ} \mathrm{C}$ using a graphite die operating in nitrogen. Some materials were also hot-pressed or annealed for a longer time in an attempt to vary the microstructure. The phase compositions were analyzed by X-ray diffraction. For a summary of the preparation conditions and the phase assemblage, see Table I, which presents the materials designation used in the rest of this paper. Finally, some typical micrographs of the microstructure are shown in Fig. 1. Note that in the micrograph to the right, grains almost always overlap because of their very small sizes compared to the thickness of the TEM sample. Additional information on the processing, microstructure, and phase equilibrium has been reported elsewhere. ${ }^{15}$

\section{(2) Testing}

Specimens for compression tests were cut from blanks and ground into $3 \mathrm{~mm} \times 3 \mathrm{~mm} \times 6 \mathrm{~mm}$ bars. Other dimensions and height-to-width ratios were also tested to evaluate the ef- 
Table I. Compositions, ${ }^{*}$ Heat Treatments, and Phase Assemblages of SiAlONS

\begin{tabular}{cccccc}
\hline & \multicolumn{4}{c}{ Composition } & \\
\cline { 2 - 5 } Designation $^{\dagger}$ & $\mathrm{Si}_{3} \mathrm{~N}_{4}$ & $\mathrm{AIN}$ & $\mathrm{Al}_{2} \mathrm{O}_{3}$ & $\mathrm{Y}_{2} \mathrm{O}_{3}$ & Phase assemblage \\
\hline $\mathrm{S} 0610$ & 83.73 & 7.77 & 3.73 & 3.70 & $30 \% \alpha^{\prime}+70 \% \beta^{\prime}$ \\
$\mathrm{H} 0610$ & 83.73 & 7.77 & 3.73 & 3.70 & $100 \% \beta^{\prime}$ \\
$\mathrm{S} 1510$ & 73.71 & 15.42 & 1.01 & 8.91 & $90 \%\left(\alpha+\alpha^{\prime}\right)+10 \% \beta^{\prime}+\mathrm{YAG}$ \\
$\mathrm{H} 1510$ & 73.71 & 15.42 & 1.01 & 8.91 & $>95 \% \alpha^{\prime}+\mathrm{YAG}$ \\
$\mathrm{S} 1010$ & 79.19 & 11.24 & 2.50 & 6.06 & $60 \%\left(\alpha+\alpha^{\prime}\right)+40 \% \beta^{\prime}+\mathrm{YAG}$ \\
$\mathrm{S} 1025$ & 68.50 & 14.69 & 10.70 & 6.09 & $35 \%\left(\alpha+\alpha^{\prime}\right)+65 \% \beta^{\prime}+\mathrm{YAG}$ \\
$\mathrm{S} 0633$ & 65.73 & 13.13 & 16.56 & 3.73 & $>95 \% \beta^{\prime}+\mathrm{YAG}$ \\
\hline
\end{tabular}

${ }^{*}$ Composition expressed in wt $\%{ }^{\dagger} \mathrm{S}$-hot-pressed at $1550^{\circ} \mathrm{C}$ for $20 \mathrm{~min}$; $\mathrm{H}$-hot-pressed at $1550^{\circ} \mathrm{C}$ for 20 min, followed by annealing at $1550^{\circ} \mathrm{C}$ for $100 \mathrm{~min}$.

fect of friction on compression experiments. The specimens were placed between two $\mathrm{SiC}$ platens. A piece of graphite foil was inserted between the specimen and the platen to further reduce friction. Testing was conducted in a vacuum furnace, with tungsten mesh heating elements, between $1500^{\circ}$ and $1575^{\circ} \mathrm{C}$ in high-purity nitrogen. The load frame used was operated under servohydraulic control to provide a constant true strain rate during the compression test. In general, a test was terminated after reaching a true strain of -0.5 . Under the above operating conditions, we found little barreling of the specimen after testing. In addition, the flow stress was independent of the height-to-width ratio used when the latter exceeded 2. Thus, friction was probably negligible in these experiments.

\section{Results}

A typical set of stress-strain curves at various strain rates in compression is shown in Fig. 2. All data shown here and below are in true stress, true strain, and true strain rate. Except at the lower strain rates, the flow stress is essentially constant over a large range of strain. Thus, a steady state is no doubt reached in those cases. The strain hardening observed at the lower strain rates is a result of microstructural coarsening. Such a phenomenon is common among fine-grained materials that deform via diffusional creep, superplasticity, or solutional reprecipitation creep, which is believed to be operational here. The initial transient reflects some elastic deformation of the specimen, the compliance of the loading train, and mostly plastic deformation during the evolution of the ini- tial microstructure. The nominal strain of the transient varies from $3 \%$ to $15 \%$, increasing with strain rate.

Comparing the flow stresses at the four strain rates in Fig. 2 at the same amount of time, say $500 \mathrm{~s}$ after the start of deformation, we find that the ratio of the two lowest flow stresses $(2.0)$ is the same as that of the corresponding strain rates, but the ratio of the two highest flow stresses (1.9) is much higher than that of the corresponding strain rates (1.4). In rheological terms, then, deformation at the two lower strain rates is Newtonian, but deformation at the higher strain rates is shear thickening. If the flow stress data are read at the same strain, say 0.15 , then we still find shear thickening at the higher strain rates, but shear thinning instead at the lower strain rates.

Data of flow stress and strain rate have been collected over a wide range of deformation conditions for a large number of superplastic SiAION materials. (In the case of continuous strain hardening, the flow stress data are ones obtained at $500 \mathrm{~s}$ after deformation. Otherwise, the steady-state flow stress data are used.) Some representative ones are shown in Fig. 3. The data shown in this figure were obtained at one test temperature, $1550^{\circ} \mathrm{C}$, and are expressed in stress versus strain rate, both in logarithmic scale. They all have a rather abrupt change in the slope, which is the same as the reciprocal stress exponent, $1 / n$ (also commonly termed strain rate sensitivity, $m, m=(\partial \ln \sigma / \partial \ln \dot{\varepsilon})$, especially in the superplasticity literature). It has a value of unity below the transition stress, and greater than unity above that. This corresponds to $n=1$ below the transition and $n \sim 0.5$ above the transition. It is important to note that, despite the wide range of strain rate

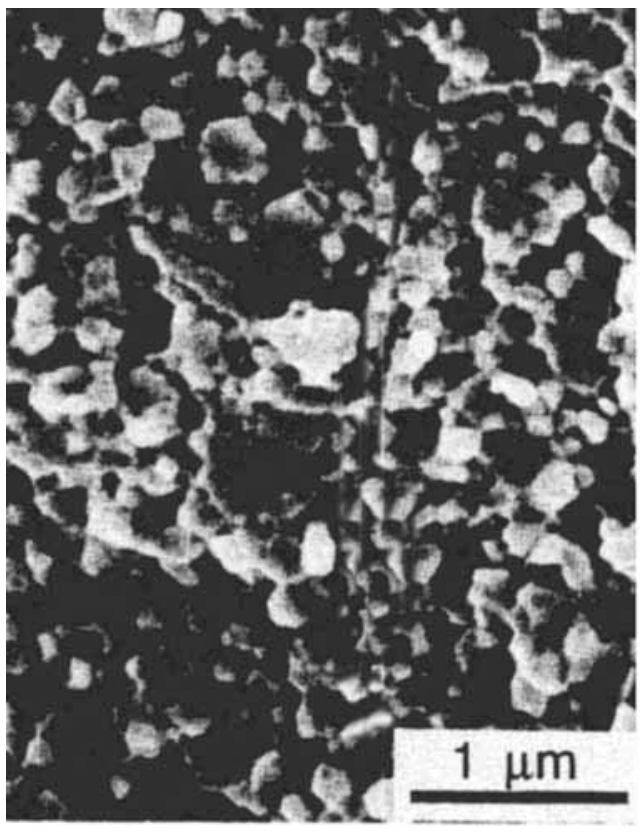

(A)

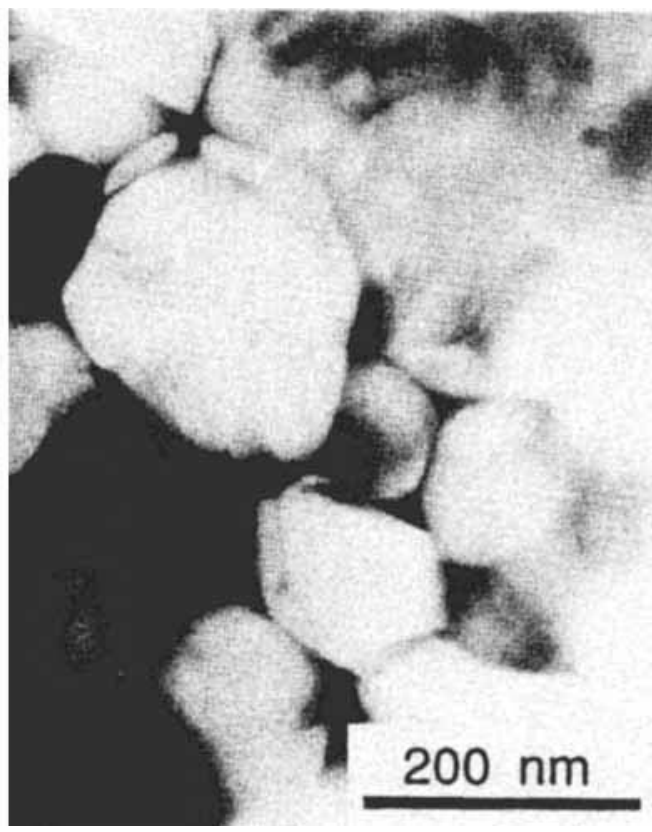

(B)

Fig. 1. Microstructure of superplastic SiAION S0610: (A) SEM, (B) TEM. 


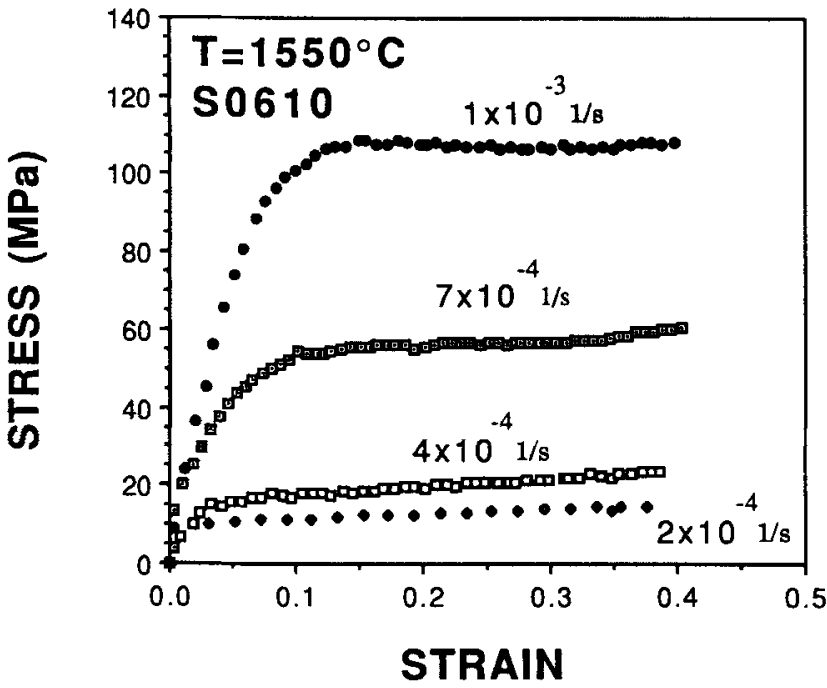

Fig. 2. Compressive stress-strain curves at various strain rates of

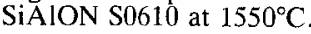

covered, the transition stress always lies near $20 \mathrm{MPa}$. Data of one material at different temperatures from $1500^{\circ}$ to $1575^{\circ} \mathrm{C}$ are shown in Fig. 4. A similar transition, occurring at approximately the same stress, is seen in every case. Moreover, the slopes below and above the transition are the same as those found in Fig. 3. In short, flow in uniaxial compression at stresses higher than the transition stress, now termed $\sigma^{*}$, is much more sensitive to strain rate than is Newtonian flow, requiring a much higher flow stress.

Transient deformation experiments involving a rate increase or rate decrease have also been performed. The rate increase in equal increments was programmed to occur at equal strain increments up to a maximum strain rate and then similarly decreased again to zero. The recorded flow stress just prior to the step increase/decrease is shown as a data point in Fig. 5, and the trajectory that connects all the data points forms a stress-strain-rate loop. In this plot we found a hysteresis (in the counterclockwise sense) in the shear-thickening regime, which is absent in the Newtonian regime. The latter hysteresis indicates that, after a strain rate decrease, it requires considerable time (strain) to fully relax the flow stress to the steady-state value. Despite the hysteresis, the transition is, nevertheless, reversible in either direction. This kind of transient, termed rheopexy, is common among fluids which are shear thickening. ${ }^{3,16}$ For shear-thinning fluids, the loop is of

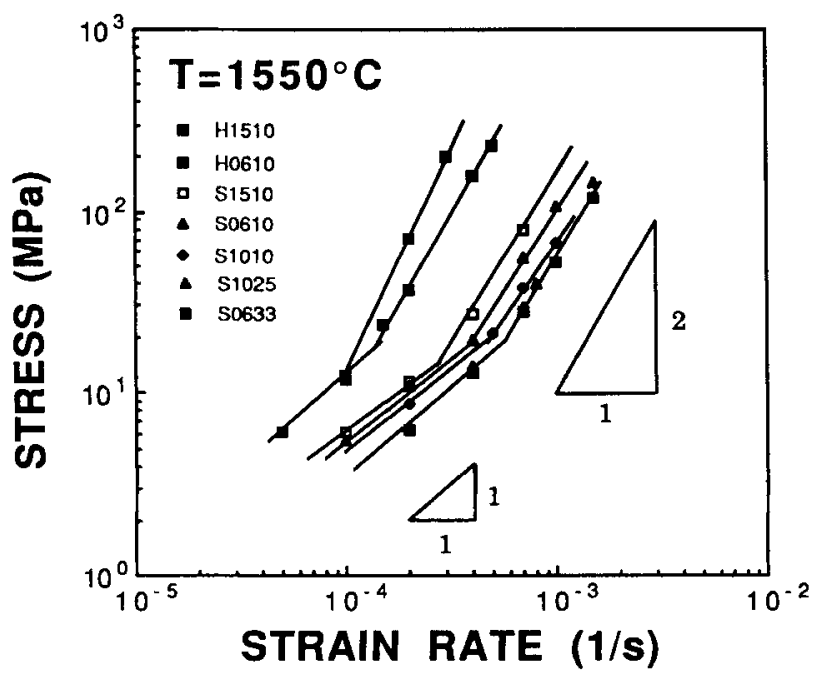

Fig. 3. Stress versus strain rate at $1550^{\circ} \mathrm{C}$ for several superplastic SiAlONs in compression $(m=$ slope $=(\partial \ln \sigma) /(\partial \ln \dot{\varepsilon}))$.

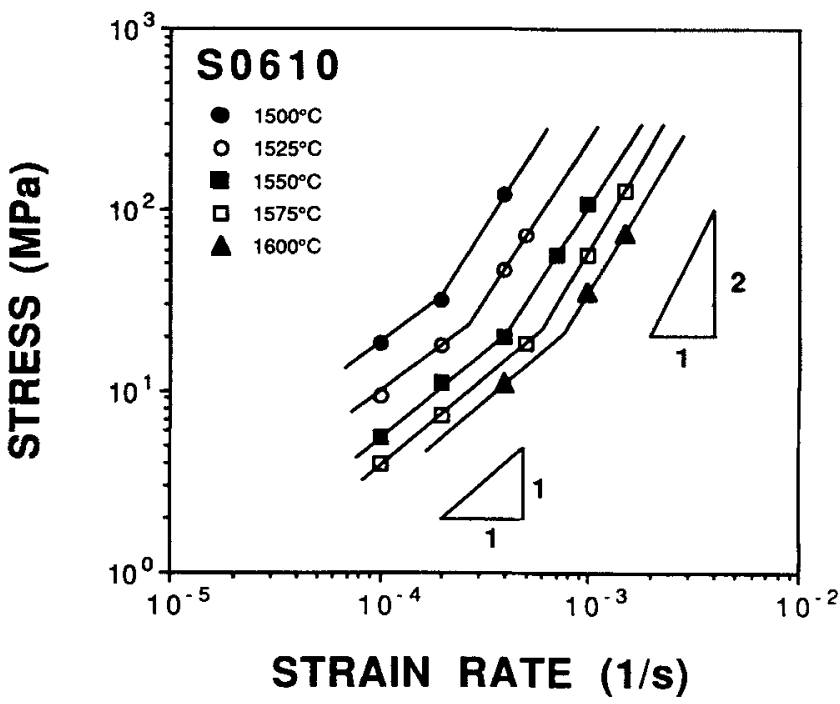

Fig. 4. Stress versus strain rate for $\mathrm{S} 0610 \mathrm{SiAlON}$ at various temperatures in compression $(m=$ slope $=(\partial \ln \sigma) /(\partial \ln \dot{\varepsilon}))$

the opposite nature (clockwise). ${ }^{3,16}$ (For solids undergoing dislocation creep, a similar transient reflecting the evolution of internal stresses has also been observed, even though $n>1$ in this case. ${ }^{17}$

Lastly, we have tried, but failed, to verify a similar Newtonian shear-thickening transition in tensile deformation. Although very large elongation can be obtained at lower stresses in many SiAION materials studied here, none has a steadystate tensile flow stress high enough in comparison with compression deformation in the shear-thickening regime. A more complete report of the constitutive region of superplastic flow of these SiAlONs, along with microstructural studies, has been provided elsewhere. ${ }^{15}$

\section{Model for Newtonian-Shear-Thickening Transition}

Let us now summarize our observations regarding the Newtonian-shear-thickening transition. The transition occurs at a characteristic stress, of the order of $20 \mathrm{MPa}$, which is independent of temperature and phase composition. The transition is reversible and strain independent but has a hysteresis in the shear-thickening regime. Although the data are not presented here, we have also found that creep in the Newtonian game is grain size dependent, which is responsible for the

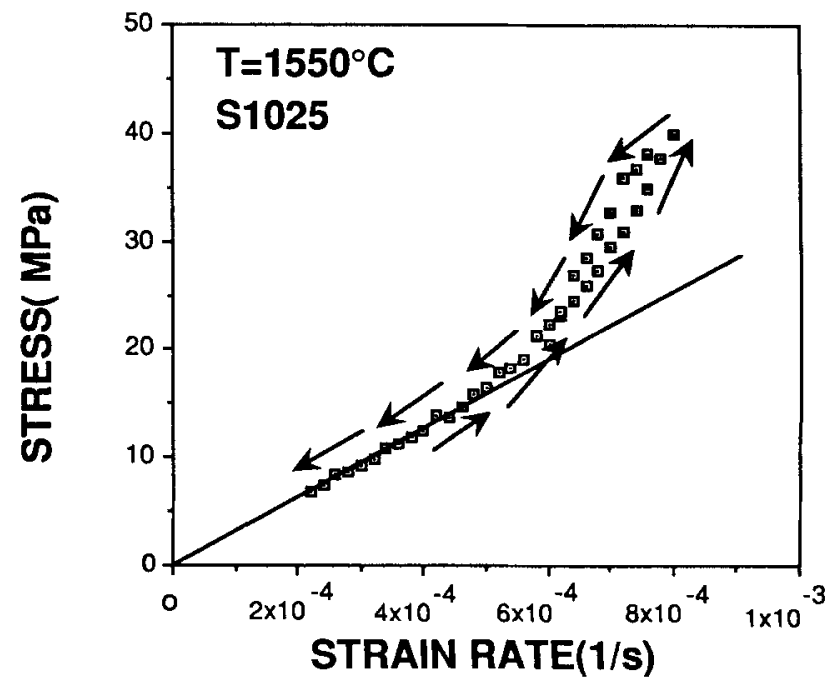

Fig. 5. Transient stress-strain rate loop of $\$ 1025$ SiAlON tested at $1550^{\circ} \mathrm{C}$ in compression. 
strain hardening seen in Fig. 2 at the lowest strain rate. ${ }^{15}$ Indeed, it must be operated by a diffusion-related mechanism, such as diffusional creep (most likely enhanced by the liquid phase) or solution/reprecipitation creep, ${ }^{18-22}$ in conjunction with grain-boundary sliding, rotation, and switching events which continually stimulate the evolution of the grain/grain configurations to sustain large strain deformation. The latter are evidently needed for steady-state superplastic flow. ${ }^{23}$

We now formulate a continuum mechanics description to model the above transition. We first postulate that, in the SiAlON materials, a micromechanical transition from a deformable Newtonian state to a nondeformable rigid state can occur within isolated volume elements at stresses above $\sigma^{*}$. Let the volume fraction of the rigid state "phase" be $\nu_{\mathfrak{t}}$, and the creep rate of the Newtonian state phase be $\dot{\varepsilon}_{0}$; then

$$
\dot{\varepsilon}_{0}=\sigma / \eta
$$

where $\eta$ is an apparent viscosity which is dependent on temperature, grain size, phase composition, and liquid content. The creep rate of the "composite" of the two "phases" is given by

$$
\dot{\varepsilon}=\left(1-\nu_{\mathrm{f}}\right)^{2.5} \dot{\varepsilon}_{0}
$$

In the above, the result of a recent study by Yoon and Chen ${ }^{24}$ on a superplastic flow of a two-phase composite containing rigid inclusions in a soft matrix was used to obtain the prefactor on the right-hand side. If we further envision that $\nu_{\mathrm{f}}$ increases with stress above $\sigma^{*}$, then the transition from Newtonian flow to shear-thickening flow can be rationalized.

To develop the model further, we next associate the unit of volume to a small region containing one grain boundary only. We also propose that, when the normal pressure on the grain boundary exceeds a certain critical stress $\sigma_{\mathrm{c}}$, the associated volume element becomes rigid. Thus, the volume fraction of the rigid "phase" $\nu_{\mathrm{f}}$ is simply the fraction of grain boundaries that support a normal pressure in excess of $\sigma_{\mathrm{c}}$.

The average normal pressure on grain boundaries can be estimated approximately using a two-dimensional picture. Grain boundaries are pictured, as in Fig. 6, to be randomly oriented spatially, but with a locally equillibrated configuration containing segments intersecting at $120^{\circ}$ with each other. The average normal pressure on any such boundary of any orientation can be computed approximately by referring to a periodic array of hexagonal grains of the same orientation. This seems to be a reasonable approximation which, in effect, replaces the actual boundary condition by a periodic one at some distance from the grain boundary of interest. As shown in the Appendix, the average normal pressure $\bar{\sigma}$ thus computed may be represented as

$$
\bar{\sigma}=\sigma\left(\frac{1}{2}+\cos 2 \theta\right)
$$
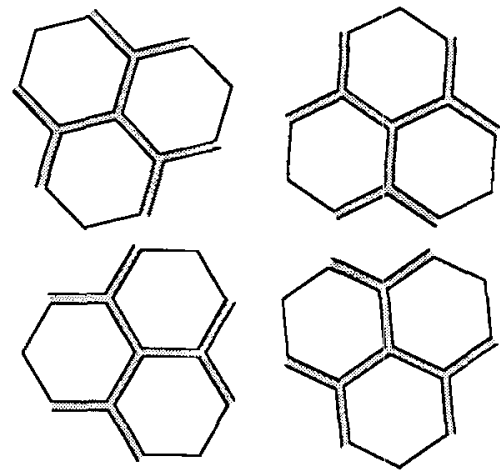

Fig. 6. Polycrystal with wetted grain boundaries at various orientations. where $\theta$ is the angle between grain-boundary normal and the external stress direction. It should be noted that the maximum normal pressure occurs at $\theta=0$, where $\bar{\sigma}=1.5 \sigma$. For $90^{\circ} \geq \theta \geq 60^{\circ}$, a negative normal pressure, i.e., tension, should prevail. In the following, we will assume that the above stress estimate for hexagonal grains is also applicable in the three-dimensional case for evaluating rigid grain boundaries.

As the applied stress in uniaxial compression increases to reach $\frac{2}{3} \sigma_{\mathrm{c}}$, grain boundaries which are normal to the stress axis $(\theta=0)$ first become rigid. As the stress increases further, some grain boundaries with nonzero $\theta$ angle also become rigid. The fraction of such rigid grain boundaries is found by evaluating the solid angle of all the boundaries in three dimensions up to an azimuthal angle $\theta$ from the pole, normalized by $4 \pi$

$$
\nu_{\mathrm{f}}=\frac{1}{4 \pi} \int_{0}^{2 \pi} \int_{0}^{1 / 2 \cos ^{-1}\left(\frac{\sigma_{\mathrm{c}}}{\sigma}-\frac{1}{2}\right)} 2 \sin \theta \mathrm{d} \theta \mathrm{d} \phi \quad\left(\sigma \geq \frac{2}{3} \sigma_{c}\right)
$$

In the above, the factor 2 in the integrand is introduced to account for symmetry (between $\theta$ and $180^{\circ}-\theta$ ), and the upper integration limit for $\theta$ is set to coincide with $\bar{\sigma}=\sigma_{c}$. Evaluating the integration and after some trigonometric manipulation, we obtain

$$
\nu_{\mathrm{f}}=1-\sqrt{\frac{1}{4}+\frac{\sigma_{\mathrm{c}}}{2 \sigma}} \quad\left(\sigma \geq \frac{2}{3} \sigma_{\mathrm{c}}\right)
$$

Note that $\nu_{\mathrm{f}}$ is always higher than 0.5 , since grain boundaries with $90^{\circ} \geq \theta \geq 60^{\circ}$ are not in compression. Combining Eqs. (6) and $(3)$, this yields, finally, the strain rate

$$
\dot{\varepsilon}=\left\{\begin{array}{ll}
\sigma / \eta & \left(\sigma<\frac{2}{3} \sigma_{c}\right) \\
\left(\frac{1}{4}+\frac{\sigma_{c}}{2 \sigma}\right)^{1.25} \frac{\sigma}{\eta} & \left(\sigma \geq \frac{2}{3} \sigma_{c}\right)
\end{array}\right\}
$$

The above results are plotted in Fig. 7 in $\log \sigma-\log \dot{\varepsilon}$ to compare with the experimental data shown in Figs. 3 and 4 . If we identify $\sigma^{*}$ with $\frac{2}{3} \sigma_{c}$, then the shape of the predicted $\sigma-\dot{\varepsilon}$ plot, including its slope in the two regimes, is similar to the one observed. The reason that the predicted transition looks

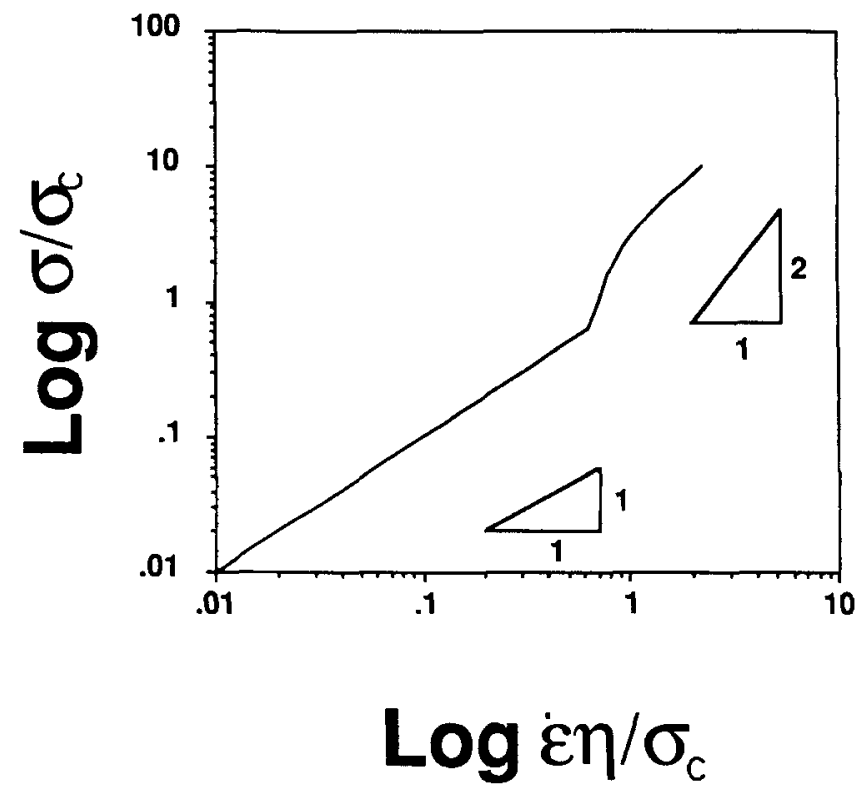

Fig. 7. Predicted stress-strain rate curve based on a two-phase model. 
sharper is that we have assumed an abrupt transition at the critical stress $\sigma_{\mathfrak{c}}$. It could have been smoothed out by allowing some distribution in grain size, grain shape, etc. Overall, then, the mechanical model seems to capture the basic characteristics of the Newtonian/shear-thickening transition.

The physical origin of the critical stress, $\sigma_{c}$, which is responsible locally for the transition, remains to be identified. This will be discussed in the next section.

\section{Discussion}

\section{(1) Grain/Liquid/Grain Interaction}

It is now well-known that almost all grain boundaries in silicon nitrides and SiAlONs are wetted by a thin glassy phase. ${ }^{25}$ Clarke $^{26}$ has argued that an equilibrium thickness (denoted as $\Delta_{0}$ ) of this phase should be maintained because the van der Waal attraction between grains is counterbalanced by a repulsive interaction due to the structure of the liquid. His model predicts an interaction potential similar to that given by the Dejaguin-Landau-Verwey-Overbeek (DLVO) theory of colloidal stability where a balance is maintained between attractive van der Waal forces and repulsive electrostatic forces. ${ }^{27}$ Plotting this potential as a function of grain/ grain separation, $h$, a primary minimum at small $h$ with a very high potential barrier outside and a shallow secondary minimum further beyond that is the common feature predicted by this model. Thus, a stress-free grain boundary should maintain an intergranular glassy phase of an equilibrium thickness $\Delta_{0}$, located at the secondary minimum, while a stressed grain boundary will become slightly depressed toward the potential barrier but otherwise able to support a normal pressure, provided the potential barrier outside the primary minimum is not exceeded.

Our experimental results are consistent with the idea that a repulsive force between grains exists: at pressures above $\sigma_{c}$, grain boundaries seem to be able to support a large normal pressure without much further deformation. On the other hand, since the Newtonian/shear-thickening transition is reversible, it may be further concluded that the potential barrier is quite high, enough to support several hundred $\mathrm{MPa}$ as seen from Figs. 2 and 3 so that the primary minimum where reversibility would have been lost is not accessible in our experiments.

One unsatisfactory aspect of the above model is that it leaves the significance of $\sigma_{\mathrm{c}}$, and the appearance of a sharp transition stress $\sigma^{*}$, unclarified. In our opinion, this can be readily improved by incorporating the concept of a Stern layer at the liquid/grain interface, again in analogy to the theory of colloidal stability. ${ }^{27}$ As shown in Fig. 8 , if a Stern layer of compactly packed liquid molecules forms on the interface, then the grain/grain interaction given by Clarke's model is truncated at twice the distance of one Stern layer thickness $2 \Delta_{\mathrm{s}}$, and the grain-to-grain distance should be limited to the same. For the same potential between "bare" grains, the truncated potential at $2 \Delta_{\mathrm{s}}$ decreases with increasing $\Delta_{5}$. Using the above picture, we interpret that, in the SiAlON ceramics studied here, the Stern layer is sufficiently thick to render only a relatively low repulsion remaining at $\Delta_{0} \geq h \geq 2 \Delta_{\mathrm{s}}$. Thus, the flow behavior is virtually unaffected by the grain/liquid/grain interaction until the normal pressure is sufficient to squeeze out the liquid so that only the Stern layers remain. This would occur at $\sigma^{*}=20 \mathrm{MPa}$, or through our model which established its relation to $\sigma_{c}$, at a repulsive force of $30 \mathrm{MPa}$ or so. The latter stress is then the repulsion at $h=2 \Delta_{\mathrm{s}}$ in the SiAION system. In view of the compactness of the Stern layer structure compared to a normal liquid, we further suggest that, at stresses above $\sigma_{c}$, with the grain-boundary liquid entirely squeezed out except for the Stern layers, both grainboundary sliding and grain-boundary diffusion would be much retarded. It is in this sense that the grain boundary has become "rigid" and "dry," and the micromechanical transition envisioned in Section IV is realized.

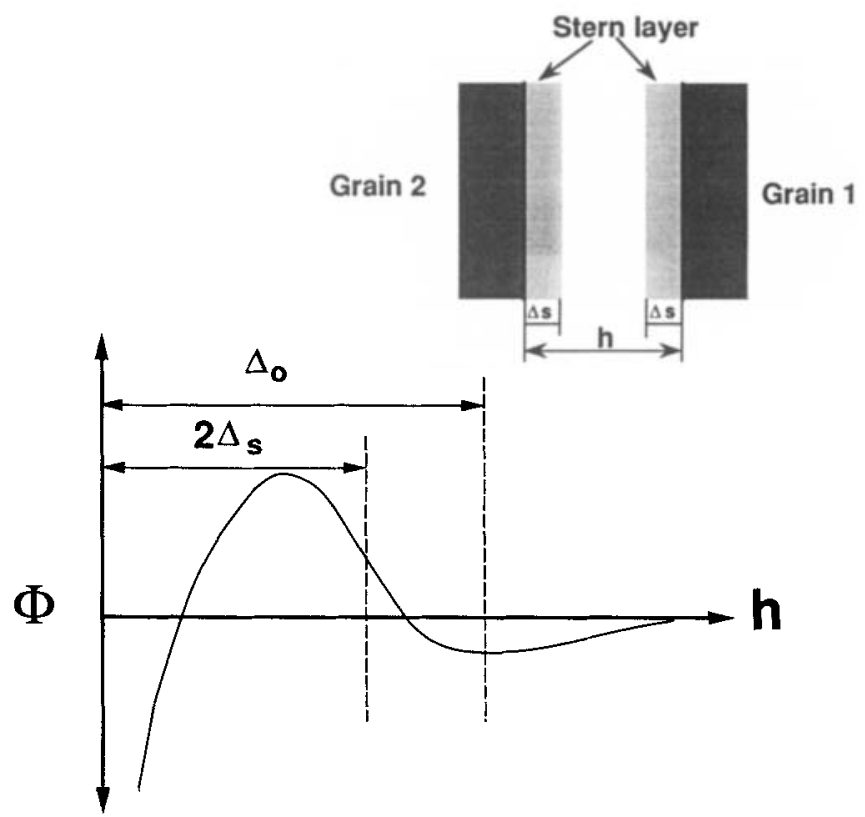

Fig. 8. Interaction $\phi$ between grains at a separation $h$, with two Stern layers on the grain/liquid interfaces.

A rough calculation is now made to estimate the critical stress $\sigma_{\mathrm{c}}$, using Clarke's model. At a distance greater than $2 \Delta_{\mathrm{s}}$, the normal stress between grains separated by a liquid can be expressed as

$$
F=\frac{H}{6 \pi h^{3}}-1.6 \times 10^{9}\left(\mathrm{~J} / \mathrm{m}^{2}\right) \eta_{0}^{2} \exp \left(\frac{-h}{\xi}\right)
$$

(At a distance smaller than $2 \Delta_{5}$, the Stern layers are in touch and we expect the repulsion to increase rapidly beyond that given by Eq. (8).) In the above, $H$ is the Hamaker constant, $\eta_{0}$ is the ordering parameter $\left(0 \leq \eta_{0} \leq 1\right)$, and $\xi$ is the correlation length for the liquid. Following Clarke, we choose $\xi=$ $0.3 \mathrm{~nm}$, which is the molecular size of the $\mathrm{SiO}_{4}$ tetrahedral unit representative of the grain-boundary liquid, and $H=$ $7.6 \times 10^{-20} \mathrm{~J}$ for $\left(\mathrm{Si}_{3} \mathrm{~N}_{4}\right)_{s}-\left(\mathrm{SiO}_{2}\right)_{I}-\left(\mathrm{Si}_{3} \mathrm{~N}_{4}\right)_{s}$ solid-liquidsolid combination. If we further assume that the thickness of the Stern layer is the same as $\xi$, then the estimated stress at $h=2 \Delta_{\mathrm{s}}=2 \xi$ is $35 \mathrm{MPa}$ (for $\eta_{0}=0.5$ ), which is very close, perhaps fortuitously, to our measured $\sigma_{c}$ value of $30 \mathrm{MPa}$.

\section{(2) Shear-Thickening Models and Observations}

Several other interpretations of the shear-thickening phenomenon in slurries, colloids, and molecular melts or solutions were mentioned in the Introduction. The models proposed for polymeric melts and solutions ${ }^{3-5,28,29}$ envision flow-induced association, entanglement, or crystallization of molecules, due to enhanced collisions, chain alignment, or chain stretching. These ideas are quite specific to polymeric molecular structures and generally not applicable to the other cases, although the notion that two or more molecules can form "quasi aggregates" $" 30.31$ which have a higher viscosity than the unassociated polymeric solutions is certainly analogous to our micromechanical picture of "rigid" inclusions. The idea of network formation under enhanced collision kinetics can be extended to colloids in which a gellike behavior with some dilatancy prior to a steep viscosity increase has been observed. ${ }^{3}$ In this case, the initial state contains colloidal particles that are separated by repulsion which, under the flow action, are brought into contact (i.e., falling into the primary minimum). Such a gellike transition, however, is often irreversible, as manifested by a permanent increase in the sedimentation volume. This is in contrast to the reversible transition described in the previous section. It has also been established for colloids that, under a shear stress, colloidal particles are organized into closely 
packed layers by hydrodynamic action to minimize the resistance to shear, in a manner not unlike the slip pattern in crystalline solids. ${ }^{8,9}$ At a certain shear stress, flow instability occurs when particles fall between layers, resulting in a flow "jam" and a rapid increase of viscosity. This instability stress has been found to be highly sensitive to the size and volume fraction of the particles, as well as to their size and shape distribution. ${ }^{8}$ This latter feature is contradictory to our observation of a constant characteristic stress at the transition.

Besides the above interpretations, there is the classical model for shear thickening based on the observation in sand and soil. Essentially, in a closely packed slurry, such as wet sand, particle rolling will inevitably cause a volumetric dilation that draws in liquid, as originally commented on by Reynolds. ${ }^{10}$ If there is just enough liquid present initially to wet all the closely packed particles, any disturbance of this configuration is likely to generate some open pores. The shear-thickening transition may then be associated with the liquid breakup (i.e., cavitation) that produces localized dewetted regions adjacent to liquid-filled pores, with local drying being responsible for the increase in flow resistance. "Such a transition is expected to be dictated by the capillarity force of the pores which must scale, by similitude, with reciprocal particle size. This prediction is again contradictory to our observation of a constant transition stress over the varied microstructural length scales investigated. We should acknowledge, of course, that our model also envisions a liquid redistribution and some "drying" (albeit with the Stern layer remaining) even though no actual dewetting/cavitation is expected.

Finally, it should be emphasized that all shear thickening reported for the above systems was obtained at a much higher strain rate, a much lower particle fraction, a much larger particle size (except perhaps in polymeric solutions), a much lower temperature, and probably with little shape accommodation of the particle itself by elastic-plastic deformation or diffusion. ${ }^{3.9 .16}$ This distinction makes the present observation rather unusual among the large variety of shear-thickening reports found in the literature. In this respect, it is not surprising to find these previous models inconsistent with our observation.

\section{(3) Concurrent and Competing Flow Mechanisms}

The flow phenomena described in this work occurred in steady-state deformation at large strains. A continual evolution of grain/grain configurations and the interchange of mechanical states are essential for any large-strain, steady-state, two-phase flow process, especially if one of the constituent phases is nondeformable. Thus, the grain boundaries which become rigid do so only for a certain time, and they are eventually relieved from pressure and separated; meanwhile, grains at other locations are compressed to become rigid. Presumably, these changes are driven by stochastic grainboundary sliding and grain rotation events. ${ }^{32}$ Without this evolution and interchange of states, grains subject to a high normal pressure would have been locked and the creep process would have been stopped. Indeed, such an exhausting creep process involving depletion of a viscous liquid on highly pressed grain boundaries has been recently described by several investigators. ${ }^{33.34}$ If maximal strains before exhaustion in such a process are of the order of the liquid fraction, which is much less than the superplastic strain achieved in our experiments, it is clear that shear thickening is not directly related to the latter mechanism. On the other hand, the transient behavior described in Section III, which is probably due to the squeezing out or replenishing of the liquid as the compressed grain boundaries adjust their liquid thickness between $\Delta_{0}$ and $2 \Delta_{5}$, can be understood using similar viscous flow models. ${ }^{33-35}$

While superplastic SiAlONs may not be the only ceramics which exhibit a shear-thickening transition, such a transition could be masked by other concurrent mechanisms operating in creep. For example, creep deformation of ceramics is frequently interrupted by cavitation at grain boundaries, even in compression, due to the tensile stress concentration at triple points where incompatibility from grain-boundary sliding is most severe. ${ }^{36}$ In silicon nitride, for example, a cavitationrelated transition from $n=1$ to $n=2$ has been reported. ${ }^{37}$ Similar observations in glass-ceramics are also well documented. ${ }^{38}$ Such a transition is of the opposite type of shearthickening transition and can obscure the latter if occurring at the same time. Since the magnitude of the local tensile stress rises with the grain size in diffusional creep and the like (superplasticity and liquid-enhanced dissolution/reprecipitation), a fine-grained microstructure which has a higher ductility and lower flow stress is probably needed for avoiding cavitation and for observing shear thickening in ceramics. Shear-thickening transitions in certain systems may also be unimportant if the transition stress is too high. For example, if our proposed model proves correct, then the structure and thickness of the liquid absorption on the grain/liquid interface will dictate the transition stress, which could vary over several orders of magnitude in different systems because of the short-ranged nature of the repulsion at the scale of the coherent length. ${ }^{26}$ While further studies are required to provide a better understanding of this aspect, it seems noteworthy that shear thickening was not observed in other superplastic ceramics (zirconia, ${ }^{12,18,24}$ alumina, ${ }^{12,39}$ mullite, ${ }^{40}$ and glassceramics $^{20}$ ). If so, it may well be a unique flow behavior of SiAlONs.

\section{APPENDIX}

\section{Average Normal Stress on an Inclined Grain Boundary}

The average normal stress on any inclined grain boundary in a periodic hexagonal array can be obtained from equilibrium consideration, provided no shear traction is transmitted by grain boundaries. Consider the periodic array shown in Fig. A1 and draw the free-body diagram as outlined by the dashed lines and the two adjacent grain boundaries. The average normal stresses on the two grain boundaries are denoted

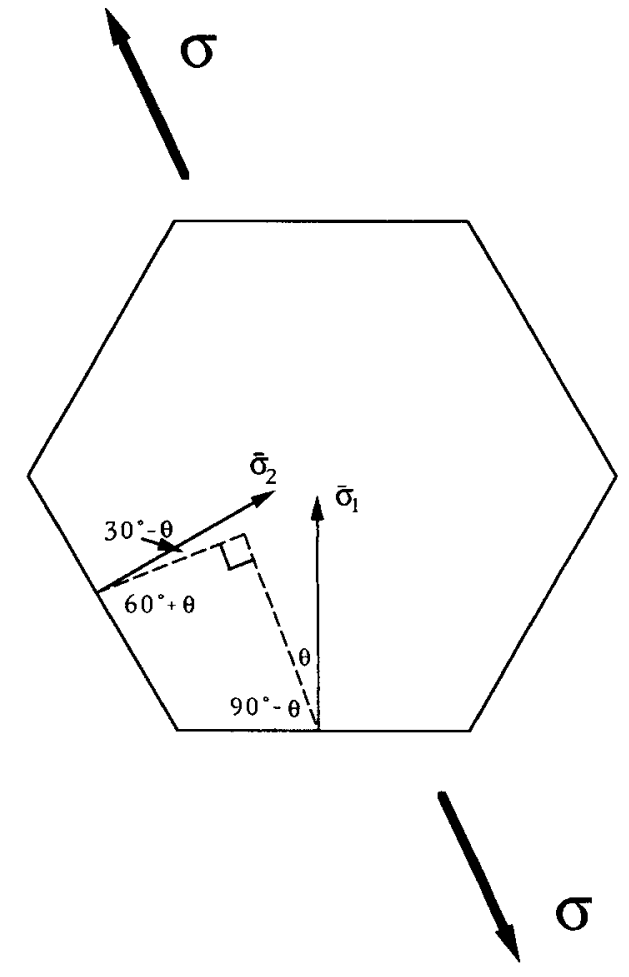

Fig. A1. Hexagonal grain in a periodic array loaded by a distant uniaxial tensile stress and locally by normal stresses on the grain boundaries. 
by $\bar{\sigma}_{1}$ and $\bar{\sigma}_{2}$. Their force balance in the applied stress direction gives

$$
\bar{\sigma}_{1} \cos \theta+\bar{\sigma}_{2} \cos \left(60^{\circ}+\theta\right)=\sigma\left[\cos \theta+\cos \left(60^{\circ}+\theta\right)\right]
$$

Similarly, force balance in the orthorgonal direction, where there is no external stress, gives

$$
\bar{\sigma}_{1} \sin \theta+\bar{\sigma}_{2} \sin \left(60^{\circ}+\theta\right)=0
$$

Solving the above set of simultaneous equations and simplifying the solution by trigonometric manipulation, we obtain the following result:

$$
\bar{\sigma}_{1} / \sigma=\frac{1}{2}+\cos 2 \theta
$$

and

$$
\bar{\sigma}_{2} / \bar{\sigma}_{1}=-\sin \theta / \sin \left(60^{\circ}+\theta\right)
$$

Of course, Eq. (A-4) can be shown to be the same as Eq. (A-3), following a transformation from $\theta$ to $60^{\circ}+\theta$. Thus, only Eq. (A-3) is quoted in the text, as Eq. (4).

The above solution is exact and independent of the actual mechanism of deformation operating in the material. The assumption of zero boundary shear traction is reasonable as long as grain-boundary sliding is not impeded.

\section{References}

${ }^{1}$ W. R. Cannon and T. G. Langdon, "Review-Creep of Ceramics, Part 1 Mechanical Characterization," J. Mater. Sci., 18, 1-50 (1983).

2W. R. Cannon and T. G. Langdon, "Review-Creep of Ceramics, Part 2 An Examination of Flow Mechanisms," J. Mater. Sci., 23, 1-20 (1988).

"W. H. Bauer and E. A. Collins, "Thixotropy and Dilatancy"; Ch. 8 , pp. 423-59 in Rheology. Theory and Applications, Vol. 4. Edited by F. R. Eirich. Academic Press, New York, 1967.

${ }^{4}$ E. P. Vrahopoulou and A. J. McHugh, "Shear-Induced Association of Polymer Molecules," Chem. Eng. Commun., 57, 289-95 (1987).

SE. P. Vrahopoulou and A. J. McHugh, "A Consideration of the Yamamoto Network Theory with Non-Gaussian Chain Segments," J. Rheol., 31 [5] 37184 (1987).

'J.Th. G. Overbeek, "Rheology of Lyophobic Systems"; Ch. 9, pp. 342-68, in Colloid Science. Edited by H. R. Kruyt. Elsevier Publishing Co., Amsterdam, Netherlands, 1952.

${ }^{7} \mathrm{H}$. van Olphen, Clay Colloid Chemistry, 2nd ed., Wiley, New York, 1977.

${ }^{8}$ R. L. Hoffman, "Discontinuous and Dilatant Viscosity Behavior in Concentrated Suspensions, II. Theory and Experimental Tests," J. Colloid Interface Sci., 46 [3] 491-506 (1974).

${ }^{9}$ R. L. Hoffman, "Discontinuous and Dilatant Viscosity Behavior in Concentrated Suspensions, III. Necessary Conditions for Their Occurrence in Viscometric Flows," Adv. Colloid. Inierface Sci., 17, 161-84 (1982).

${ }^{10} \mathrm{O}$. Reynolds, "On the Dilatancy and Media Composed of Rigid Particles in Contact," Philos. Mag., 20 [127] 469-81 (1985).

"H. Freundlich and H. L. Roder, "Dilatancy and Its Relation to Thixotropy," Trans. Faraday Soc., 34 [127] 308-16 (1938).

${ }^{12} \mathrm{I}-W$. Chen and L. A. Xue, "Development of Superplastic Structural Ceramics," J. Am. Ceram. Soc., 73 [9] 2585-609 (1990).

${ }^{13}$ L. J. Guackler, H. L. Lukas, and T.Y. Tien, "Crystal Chemistry of $\beta-\mathrm{Si}_{3} \mathrm{~N}_{4}$ Solid Solutions Containing Metal Oxides," Mater. Res, Bull., 11 [5] 503-12 (1976).

${ }^{14}$ S. Hampshire, H. K. Park, D. P. Thompson, and K. H. Jack, " $\alpha$ '-Sialon Ceramics," Nature (London), 274 [5674] 880-82 (1978).
${ }^{15}$ S. L. Hwang and I-W. Chen, "Transient-Liquid Aided Superplastic Forming of Silicon Nitrides"; presented at the 93rd Annual Meeting of the American Ceramic Society, Cincinnati, OH, April 28-May 2, 1991 (Paper No. 37SI-91).

${ }^{16}$ H. A. Barnes, "Shear-Thickening ("Dilatancy") in Suspensions of Nonaggregating Solid Particles Dispersed in Newtonian Liquids," J. Rheol., 33 [2] 329-66 (1989).

${ }^{17}$ S. Takeuchi and A.S. Argon, "Review-Steady-State Creep of SinglePhase Crystalline Matter at High Temperatures," J. Mater. Sci., 11, 1542-66 (1976).

${ }^{18}$ C.-M. J. Hwang and I-W. Chen, "Effect of a Liquid Phase on Superplasticity of 2-mol\%-Y $\mathrm{O}_{3}$-Stabilized Tetragonal Zirconia Polycrystals," J. Am. Ceram. Soc., 73 [6] 1626-32 (1990),

${ }^{19} \mathrm{R}$. Raj and C. K. Chyung, "Solution-Precipitation Creep in Glass Ceramics," Acta Metall., 29 [1] 159-66 (1981).

${ }^{2 t}$ J.-G. Wang and R. Raj, "Mechanisms of Superplastic Flow in a FineGrained Ceramic Containing Some Liquid Phase," J. Am. Ceram. Soc., 67 [6] 399-409 (1984).

${ }^{21}$ A. Tsuge, K. Nishida, and M. Tomatsu, "Effect of Crystallizing the Grain Boundary Phase on the High-Temperature Strength of Hot-Pressed $\mathrm{Si}_{3} \mathrm{~N}_{4}$ Containing $\mathrm{Y}_{2} \mathrm{O}_{3}$, , J. Am. Ceram. Soc., 58 [7-8] 323-26 (1975).

${ }^{22}$ C. F. Chen and T.Y. Tien, "High-Temperature Mechanical Properties of SiAION Ceramics: Microstructural Effect," Ceram. Eng. Sci. Proc., 8 [7-8] 778-95 (1987).

${ }^{23}$ M. F. Ashby and R. A. Verrall, "Diffusion-Accommodated Flow and Superplasticity," Acta Metall., 21, 149-63 (1973).

${ }^{24} \mathrm{C}$. K. Yoon and I-W. Chen, "Superplastic Flow of Two-Phase Ceramics Containing Rigid Inclusions-Zirconia/Mullite Composites," J. Am. Ceram. Soc., 73 [6] 1555-65 (1990).

${ }^{25}$ D. R. Clarke and G. Thomas, "Grain Boundary Phases in a Hot-Pressed MgO Fluxed Silicon Nitride," J. Am. Ceram. Soc., 60 [11-12] 491-95 (1977).

${ }^{2} \mathrm{D}$. R. Clarke, "On the Equilibrium Thickness of Intergranular Glass Phases in Ceramic Materials,"J. Am. Ceram. Soc., 70 [1] 15-22 (1987).

${ }^{27}$ A.W. Adamson, Physical Chemistry of Surfaces, 4th ed. Wiley, New York, 1982.

${ }^{28}$ O. Quadrat, "Negative Thixotropy in Polymer Solutions," Adv. Colloid. Interf. Sci., 24, 45-75 (1985).

${ }^{29}$ E. P. Vrahopoulou and A. J. McHugh, "Shear-Thickening and Structure Formation in Polymer Solutions," J. Non-Newtonian Fluid Mech., 25, 157-75 (1987).

${ }^{30} R$. Simha, "Effect of Concentration on the Viscosity of Dilute Solutions," J. Res. Natl. Bur. Stand. (U.S.), 42, 409-18 (1949).

"S. G. Weissburg, R. Simha, and S. Rothman, "Viscosity of Dilute and Moderately Concentrated Polymer Solutions," J. Res. Nat. Bur. Stand., 47, 298-314 (1951).

${ }^{32}$ I-W. Chen and A.S. Argon, "Creep Cavitation of 304 Stainless Steel," Acta Metall., 29, 1321-33 (1981).

${ }^{33}$ J. R. Dryden, D. Kucerousky, D.S. Wilkinson, and D. F. Watt, "Creep Deformation Due to a Viscous Grain Boundary Phase," Acta Metall., 37 [7] 2007-15(1989).

${ }^{34}$ K. D. Debschutz, R. Danzer, and G. Petzow, "Finite Element Modelling of Ceramic Materials with a Viscous Grain Boundary Phase"; pp. 727-36 in Ceramic Today, Tomorrow's Ceramics. Edited by P. Vincenzini. Elsevier, Barking, Essex, U.K., 1991.

${ }^{35}$ D. C. Drucker, "Engineering and Continuum Aspects of High Strength Materials"; Ch. 27, pp. 795-833 in High-Strength Materials. Edited by V. F. Zackay. Wiley, New York, 1965.

${ }^{36}$ A. S. Argon, I-W. Chen, and C.W. Lau, "Intergranular Cavitation in Creep-Theory and Experiment"; pp. 46-85 in Creep-Fatigue-Environment Interactions. Edited by R. M. N. Pelloor and N. S. Stolloff. The Metallurgical Society of AIME, New York, 1980.

${ }^{37}$ F. F. Lange, B. I. Davis, and D. R. Clarke, "Compressive Creep of $\mathrm{Si}_{3} \mathrm{~N}_{4} / \mathrm{MgO}$ Alloys Part I. Effect of Composites," J. Mater. Sci., IS [3] 601-10 (1980).

${ }^{38} \mathrm{R}$. Morrell and K. H. G. Ashbee, "High Temperature Creep of Lithium Zinc Silicate Glass Ceramics," J. Mater. Sci., 8, 1253-70 (1973).

${ }^{39}$ L. A. Xue and I-W. Chen, "Superplastic Alumina Ceramics with Grain Growth Inhibitors," J. Am. Ceram. Soc., 74 [4] 842-45 (1991).

${ }^{40}$ L. A. Xue and I-W. Chen, "Superplastic Mullite by Transient-Phase Processing," J. Am. Ceram. Soc., in review. 